\title{
New proof to Somos's Dedekind eta-function identities of level 10
}

\author{
B. R. Srivatsa Kumar ${ }^{1}$. Shruthi ${ }^{1}$
}

Accepted: 26 April 2021 / Published online: 9 May 2021

(c) The Author(s) 2021

\begin{abstract}
Michael Somos used PARI/GP script to generate several Dedekind eta-function identities by using computer. In the present work, we prove two new Dedekind eta-function identities of level 10 discovered by Somos in two different methods. Also during this process, we give an alternate method to Somos's Dedekind eta-function identities of level 10 proved by B. R. Srivatsa Kumar and D. Anu Radha. As an application of this, we establish colored partition identities.
\end{abstract}

Keywords Dedekind eta-functions · Modular equations $\cdot$ Colored partitions

\section{Introduction}

The Dedekind eta-function $\eta(\tau)$ is defined by the formula

$\eta(\tau)=q^{1 / 24} \prod_{n=1}^{\infty}\left(1-q^{n}\right), \quad q=e^{2 \pi i \tau}$

where $\tau$ belongs to the upper complex half-plane. Here and all through the paper, we assume $|q|<1$ and employ the standard notation

$(a ; q)_{\infty}:=\prod_{n=0}^{\infty}\left(1-a q^{n}\right)$.

For $|x y|<1$, Ramanujan's theta function $\mathfrak{f}(x, y)$ is defined as

$\mathfrak{f}(x, y):=\sum_{n=-\infty}^{\infty} x^{n(n+1) / 2} y^{n(n-1) / 2}$.

Also in Ramanujan's notation, Jacobi's triple-product identity (Berndt 1991, p. 35) is given by

$\mathfrak{f}(x, y)=(-x ; x y)_{\infty}(-y ; x y)_{\infty}(x y ; x y)_{\infty}$.

Shruthi

shruthikarranth@gmail.com

B. R. Srivatsa Kumar

sri_vatsabr@yahoo.com

1 Department of Mathematics, Manipal Institute of Technology, Manipal Academy of Higher Education, 576104 Manipal, India
The important special cases of $\mathfrak{f}(x, y)$ (Berndt 1991, p. 36) are

$$
\begin{aligned}
\varphi(q) & :=\mathfrak{f}(q, q)=\sum_{n=-\infty}^{\infty} q^{n^{2}}=\left(-q ; q^{2}\right)_{\infty}^{2}\left(q^{2} ; q^{2}\right)_{\infty}, \\
\psi(q) & :=\mathfrak{f}\left(q, q^{3}\right)=\sum_{n=0}^{\infty} q^{\frac{n(n+1)}{2}}=\frac{\left(q^{2} ; q^{2}\right)_{\infty}}{\left(q ; q^{2}\right)_{\infty}}, \\
f(-q) & :=\mathfrak{f}\left(-q,-q^{2}\right) \\
& =\sum_{n=-\infty}^{\infty}(-1)^{n} q^{n(3 n-1) / 2}=(q ; q)_{\infty} .
\end{aligned}
$$

Following Ramanujan's notations, for $q=e^{2 \pi i \tau}$, we set

$f(-q)=q^{-1 / 24} \eta(\tau)$

Also after Ramanujan, define

$\chi(q):=\left(-q ; q^{2}\right)_{\infty}$

For convenience, we write $f_{n}=f\left(-q^{n}\right)$. A theta function identity which relates $f_{1}, f_{2}, f_{n}$ and $f_{2 n}$ is called the theta function identity of level $2 n$. Ramanujan documented many modular equations which involve quotients of the function $f_{1}$ at different arguments. For example, if (Berndt 1996, p. 206) 
$P:=\frac{f_{1}}{q^{1 / 6} f_{5}} \quad$ and $\quad Q:=\frac{f_{2}}{q^{1 / 3} f_{10}}$,

then

$P Q+\frac{5}{P Q}=\left(\frac{Q}{P}\right)^{3}+\left(\frac{P}{Q}\right)^{3}$,

and if (Ramanujan 1988, p. 55)

$A:=\frac{f_{1}}{q^{1 / 24} f_{2}} \quad$ and $\quad B:=\frac{f_{5}}{q^{5 / 24} f_{10}}$,

then

$(A B)^{2}+\frac{4}{(A B)^{2}}=\left(\frac{B}{A}\right)^{3}-\left(\frac{A}{B}\right)^{3}$.

After the publication of Berndt (1991), many authors including (Adiga et al. 2002, 2004, 2016; Baruah 2002, 2003; Naika 2006; Saikia 2011; Vasuki and Sreeramamurthy 2005; Vasuki 2006; Vasuki and Veeresha 2017; Yi 2004) and many more mathematicians have found additional modular equations of the type (1) and (2) of different levels for the evaluation of various continued fractions, Weber-class invariants, two-parameter evaluation of theta functions and many more. Motivated by the above work, M. Somos [13] used a computer to discover around 6277 new elegant Dedekind eta-function identities of various levels without offering the proof. He runs PARI/GP scripts and it works as a sophisticated programmable calculator. Yuttanan (2012), Vasuki and Veeresha (2017), Srivatsa Kumar and Anu Radha (2018) and Srivatsa kumar et al. (2019) have obtained the proofs for the levels 4, 6, 8, 10, 12 and 14. Cao (2010) has given the proof of Somos's dissection identities.

Motivated by the above, in the present work we prove two new Dedekind eta-function identities in two different methods. Furthermore, we are giving a new proof for Dedekind eta-function identities of level 10 which was proved in Srivatsa Kumar and Anu Radha (2018). As an application of this, we establish the colored partition identities for the same. Now we list some of the Somos's identities of level 10:

$$
\begin{aligned}
& \frac{\varphi^{2}(-q)}{q \chi(-q) \chi\left(-q^{5}\right) \psi^{2}\left(q^{5}\right)}+20 q \frac{\chi(-q) \chi\left(-q^{5}\right) \psi^{2}\left(q^{5}\right)}{\varphi^{2}(-q)} \\
& -\frac{\chi^{6}\left(-q^{5}\right)}{q \chi^{6}(-q)}+9=0 .
\end{aligned}
$$

$$
\begin{aligned}
& \frac{4 \chi(-q) \chi\left(-q^{5}\right) \psi^{2}(q)}{\psi^{2}\left(-q^{5}\right)} \\
& +\frac{5 \varphi^{2}\left(-q^{5}\right)}{\chi(-q) \chi\left(-q^{5}\right) \psi^{2}(q)}+q \frac{\chi^{6}(-q)}{\chi^{6}\left(-q^{5}\right)}-9=0 \\
& f_{1} f_{2}^{6} f_{5}^{7}+q f_{1}^{7} f_{5} f_{10}^{6}+5 q^{2} f_{1}^{4} f_{2} f_{10}^{9}-f_{2}^{9} f_{5}^{4} f_{10}=0 \\
& f_{1}^{4} f_{5}^{4} f_{10}^{2}+16 q f_{2}^{4} f_{10}^{6}+4 f_{1}^{3} f_{2}^{3} f_{5} f_{10}^{3}-5 f_{2}^{2} f_{5}^{8}=0 \\
& 5 f_{1}^{4} f_{2} f_{10}^{2}+16 q f_{2}^{5} f_{5} f_{10}^{6}+4 q f_{1}^{5} f_{10}^{7}-5 f_{2}^{3} f_{5}^{9}=0 . \\
& q f_{2}^{4} f_{5}^{2} f_{10}^{4}+f_{1}^{4} f_{5}^{6}-f_{1}^{3} f_{2}^{3} f_{5}^{3} f_{10}-5 q^{2} f_{1}^{2} f_{10}^{8}=0 . \\
& f_{1}^{8} f_{10}^{4}+20 q f_{1} f_{2}^{3} f_{5}^{3} f_{10}^{5}+4 f_{1}^{3} f_{2}^{5} f_{5} f_{10}^{3}-5 f_{2}^{4} f_{5}^{8}=0 . \\
& f_{2}^{6} f_{5}^{6}+q f_{1}^{6} f_{10}^{6}-f_{1}^{4} f_{2}^{4} f_{5}^{2} f_{10}^{2}-5 q f_{1}^{2} f_{2}^{2} f_{5}^{4} f_{10}^{4}=0 . \\
& f_{1}^{10} f_{5}^{2} f_{10}^{2}+10 q f_{1}^{3} f_{2}^{3} f_{5}^{5} f_{10}^{3}-f_{2}^{10} f_{5}^{4}-15 q^{2} f_{1}^{4} f_{2}^{2} f_{10}^{8}=0 . \\
& f_{1}^{9} f_{5}^{2} f_{10}^{3}+9 q f_{1}^{2} f_{2}^{3} f_{5}^{5} f_{10}^{4}-f_{2}^{7} f_{5}^{7}-16 q^{2} f_{1}^{3} f_{2}^{2} f_{10}^{9}=0
\end{aligned}
$$

and many more. Before concluding this section, we define a modular equation as defined by Ramanujan. The Gauss ordinary hypergeometric series is defined by

${ }_{2} F_{1}(a, b ; c ; z):=\sum_{n=0}^{\infty} \frac{(a)_{n}(b)_{n}}{(c)_{n} n !} z^{n} \quad|z|<1$,

where

$(a)_{n}:=a(a+1)(a+2) \ldots(a+n-1)$.

Let $F(x)={ }_{2} F_{1}\left(\frac{1}{2}, \frac{1}{2} ; 1 ; x\right)$, then the relation between $\alpha$ and $\beta$ induced by the following equation is called modular equation of degree $n$ :

$n \frac{F(1-\alpha)}{F(\alpha)}=\frac{F(1-\beta)}{(\beta)}$.

Suppose that $y=\pi \frac{F(1-x)}{F(x)}$ and $z=F(x)$, then from (Berndt 1991, pp. 122-124, Entry 10(i) and 12(v)), we have

$\varphi\left(e^{-y}\right):=\sqrt{z}$

and

$\chi\left(e^{-y}\right):=2^{1 / 6}\left(\frac{x}{e^{y}}(1-x)\right)^{-1 / 24}$.

We also define the multiplier $m$ by

$m:=\frac{F(\alpha)}{F(\beta)}$. 


\section{Proof of Somos's identities of level 10}

Proof of (3) The following modular equations of degree 5 are recorded by Ramanujan on page 236 of his second notebook (Ramanujan 1957) and (Berndt 1991, pp. 280-288, Entry 13(ix) and (xiv)):

$$
\begin{aligned}
1 & +4^{1 / 3}\left(\frac{\beta^{5}(1-\beta)^{5}}{\alpha(1-\alpha)}\right)^{1 / 12} \\
& =\frac{m}{2}\left(1+(\alpha \beta)^{1 / 2}+\{(1-\alpha)(1-\beta)\}^{1 / 2}\right), \\
1 & +4^{1 / 3}\left(\frac{\alpha^{5}(1-\alpha)^{5}}{\beta(1-\beta)}\right)^{1 / 12} \\
& =\frac{5}{2 m}\left(1+(\alpha \beta)^{1 / 2}+\{(1-\alpha)(1-\beta)\}^{1 / 2}\right),
\end{aligned}
$$

and if

$$
\begin{aligned}
& P:=\{16 \alpha \beta(1-\alpha)(1-\beta)\}^{1 / 12} \text { and } \\
& Q:=\left\{\frac{\beta(1-\beta)}{\alpha(1-\alpha)}\right\}^{1 / 8},
\end{aligned}
$$

then

$Q+\frac{1}{Q}+2\left(P-\frac{1}{P}\right)=0$,

where $\beta$ has degree 5 over $\alpha$ and $m$ is the multiplier. From (10) and (11), we have

$$
\frac{m^{2}}{5}=\frac{1+4^{1 / 3}\left(\frac{\beta^{5}(1-\beta)^{5}}{\alpha(1-\alpha)}\right)^{1 / 12}}{1+4^{1 / 3}\left(\frac{\alpha^{5}(1-\alpha)^{5}}{\beta(1-\beta)}\right)^{1 / 12}} .
$$

For $q=e^{-y}$ from (8), we can write

$$
\begin{aligned}
\chi(q) & =2^{1 / 6}\left(\frac{q}{\alpha(1-\alpha)}\right)^{1 / 24} \text { and } \\
\chi\left(q^{5}\right) & =2^{1 / 6}\left(\frac{q^{5}}{\beta(1-\beta)}\right)^{1 / 24} .
\end{aligned}
$$

From the above, we deduce

$$
\begin{aligned}
& 2^{4 / 3} q^{2} \frac{\chi^{2}(q)}{\chi^{10}\left(q^{5}\right)}=\left(\frac{\beta^{5}(1-\beta)^{5}}{\alpha(1-\alpha)}\right)^{1 / 12} \quad \text { and } \\
& 2^{4 / 3} \frac{\chi^{2}\left(q^{5}\right)}{\chi^{10}(q)}=\left(\frac{\alpha^{5}(1-\alpha)^{5}}{\beta(1-\beta)}\right)^{1 / 12} .
\end{aligned}
$$

On using these in (13), also by making use of (9) and (7), we obtain

$$
\frac{\varphi^{4}(q)}{5 \varphi^{4}\left(q^{5}\right)}=\frac{1+4 \frac{q^{2} \chi^{2}(q)}{\chi^{10}\left(q^{5}\right)}}{1+4 \frac{\chi^{2}\left(q^{5}\right)}{\chi^{10}(q)}} .
$$

Similarly, on transcribing (12) into theta function, we obtain

$\frac{x^{3}}{y^{3}}+\frac{y^{3}}{x^{3}}+\frac{4}{x^{2} y^{2}}-x^{2} y^{2}=0$,

where

$x:=x(q)=q^{-1 / 24} \chi(q) \quad$ and

$y:=y(q)=q^{-5 / 24} \chi\left(q^{5}\right)$.

Now on multiplying (15) throughout by $\left(4 x^{12}+5 x^{10} y^{10}+\right.$ $\left.32 x^{6} y^{6}+80 x^{2} y^{2}+4 y^{12}\right)\left(x^{6}-4 x y+x^{5} y^{5}+y^{6}\right)$, we obtain

$$
\begin{gathered}
\frac{x^{20}}{y^{4}}-\frac{x^{22}}{5 y^{14}}-\frac{18 x^{16}}{5 y^{8}}-\frac{x^{10}}{5 y^{2}}-\frac{4 x^{24}}{5 y^{24}}-\frac{72 x^{18}}{5 y^{18}} \\
-\frac{168 x^{12}}{5 y^{12}}-\frac{72 x^{6}}{5 y^{6}}-\frac{16 x^{14}}{5 y^{22}} \\
-\frac{288 x^{8}}{5 y^{16}}-\frac{16 x^{2}}{5 y^{10}}+\frac{256 x^{4}}{y^{20}}-\frac{4}{5}=0,
\end{gathered}
$$

which can be rewritten as

$$
\begin{aligned}
& {\left[1+\frac{4 x^{2}}{y^{10}}+\frac{4 x^{2}}{y^{10}}\left(1+\frac{4 y^{2}}{x^{10}}\right)\right]^{2}} \\
& =\frac{1}{5}\left(\frac{y}{x^{5}}+\frac{9 x}{y^{5}}\right)^{2}\left(1+\frac{4 y^{2}}{x^{10}}\right)\left(1+\frac{4 x^{2}}{y^{10}}\right) .
\end{aligned}
$$

Employing (14) in the above, we see that

$$
1+20 \frac{x^{2}}{y^{10}} \frac{\varphi^{4}\left(q^{5}\right)}{\varphi^{4}(q)}=\left(\frac{y}{x^{5}}+\frac{9 x}{y^{5}}\right) \frac{\varphi^{2}\left(q^{5}\right)}{\varphi^{2}(q)} .
$$

Also without difficulty, we observe that

$\varphi(q)=\frac{f_{2}^{5}}{f_{1}^{2} f_{4}^{2}}, \quad \varphi(-q)=\frac{f_{1}^{2}}{f_{2}}, \quad \psi(q)=\frac{f_{2}^{2}}{f_{1}}$,

$\chi(q)=\frac{f_{2}^{2}}{f_{1} f_{4}}$ and $\chi(-q)=\frac{f_{1}}{f_{2}}$.

From (17), we have

$$
\frac{\varphi(q)}{\varphi\left(q^{5}\right)}=\frac{x^{2} f_{2}}{q^{1 / 3} y^{2} f_{10}} .
$$


Using the above in (16), we have

$1+\frac{20 q^{4 / 3}}{x^{6} y^{2}}\left(\frac{f_{10}}{f_{2}}\right)^{4}=q^{2 / 3}\left(\frac{y^{5}}{x^{9}}+\frac{9}{x^{3} y}\right)\left(\frac{f_{10}}{f_{2}}\right)^{2}$.

Letting $q \rightarrow-q$ in the above, rewriting $x(-q)$ and $y(-q)$ in terms of $f_{n}$ by employing (17) and then simplifying, we deduce the result.

Second proof of (3) On using (17) in (3) and then dividing throughout by $f_{1}^{9} f_{5}^{2} f_{10}^{3}$, we obtain

$1+20 q^{2} \frac{f_{2}^{2} f_{10}^{6}}{f_{1}^{6} f_{5}^{2}}+9 q \frac{f_{2} f_{10}^{3}}{f_{1}^{3} f_{5}}-\frac{f_{2}^{7} f_{5}^{5}}{f_{1}^{9} f_{10}^{3}}=0$.

On using $P, Q, A$ and $B$ as defined as in (1) and (2), (18) reduces to

$\frac{20}{(A B)^{4}(P Q)^{2}}+\frac{1}{(A B)^{2}}\left(\frac{9}{P Q}-\frac{Q^{5}}{P^{7}}\right)+1=0$

equivalently

$(A B)^{2}=\frac{40 P^{5}}{Q^{7}-9 P^{6} Q \pm \sqrt{\left(9 P^{6} Q-Q^{7}\right)^{2}-80 P^{12} Q^{2}}}$.

Using (19) in (2) and then factorizing, we obtain

$L(P, Q) M(P, Q)=0$,

where

$L(P, Q)=P^{6}-5 P^{2} Q^{2}-P^{4} Q^{4}+Q^{6}$

and

$$
\begin{aligned}
M(P, Q)= & 400 P^{10} Q^{2}\left(5 P^{12}-4 P^{10} Q^{4}\right. \\
& \left.-20 P^{8} Q^{2}-14 P^{6} Q^{6}+Q^{12}\right) .
\end{aligned}
$$

But $L(P, Q)$ is nothing but (1) and it verifies (3).

We omit the proof of (4), as the proof is similar to the previous one.

Proof of (5) Srivatsa Kumar and Anu Radha (2018) On dividing (5) by $f_{1} f_{2}^{6} f_{5}^{7}$, we obtain

$1+q \frac{f_{1}^{6} f_{10}^{6}}{f_{5}^{6} f_{2}^{6}}+5 q^{2} \frac{f_{1}^{3} f_{10}^{9}}{f_{2}^{5} f_{5}^{7}}-\frac{f_{2}^{3} f_{10}}{f_{1} f_{5}^{3}}=0$.
On using $P, Q, A$ and $B$ as defined as in (1) and (2), (20) becomes

$\frac{1}{(A B)^{2}}\left(\frac{5 P^{5}}{Q^{7}}-P Q\right)+\frac{P^{6}}{Q^{6}}+1=0$

equivalently

$(A B)^{2}=\frac{P Q^{8}-5 P^{5}}{Q^{7}+P^{6} Q}$.

Using (21) in (2) and then factorizing, we deduce

$L(P, Q) M(P, Q)=0$,

where

$L(P, Q)=P^{6}-5 P^{2} Q^{2}-P^{4} Q^{4}+Q^{6}$

and

$M(P, Q)=5 P^{10}+Q^{4} P^{8}+Q^{10} P^{2}+Q^{14}$.

But $L(P, Q)$ is nothing but (1), and it verifies (5).

Proof of (6) Srivatsa Kumar and Anu Radha (2018) On dividing (6) by $f_{2}^{2} f_{5}^{8}$, we obtain

$\frac{f_{1}^{4} f_{10}^{2}}{f_{5}^{4} f_{2}^{2}}+16 q \frac{f_{2}^{2} f_{10}^{6}}{f_{5}^{8}}+4 \frac{f_{1}^{3} f_{2} f_{10}^{3}}{f_{5}^{7}}-5=0$.

On using $P, Q, A$ and $B$ as defined as in (1) and (2), (22) becomes

$\frac{16 P^{4}}{Q^{2}(A B)^{4}}+\frac{4 P^{5}}{Q(A B)^{2}}+\frac{P^{4}}{Q^{2}}-5=0$

equivalently

$(A B)^{2}=\frac{8 P^{4}}{-P^{5} Q \pm \sqrt{P^{10}-4 P^{8}+20 P^{4} Q^{2}}}$.

Using (23) in (2) and then factorizing, we obtain

$L(P, Q) M(P, Q)=0$,

where

$L(P, Q)=P^{6}-5 P^{2} Q^{2}-P^{4} Q^{4}+Q^{6}$

and

$M(P, Q)=4 P^{4}\left(P^{10}-4 P^{8} Q^{4}-9 P^{4} Q^{6}-5 Q^{8}\right)$.

But $L(P, Q)$ is nothing but (1), and it verifies (6). 
Remark: Using the same method, we can prove the remaining Somos's Dedekind eta-function identities of level 10 which are listed in the previous section.

\section{Applications to colored partition}

The identities proved in Section 2 have applications to the theory of partitions. In this section, we demonstrate colored partitions for (3). Similarly for the remaining identities mentioned in section 1, we can establish the same concept. For simplicity, we adopt the standard notation

$\left(x_{1}, x_{2}, \ldots, x_{m} ; q\right)_{\infty}:=\prod_{j=1}^{m}\left(x_{j} ; q\right)_{\infty}$.

"A positive integer $n$ has $l$ colors if there are $l$ copies of $n$ available colors and all of them are viewed as distinct objects. Partitions of a positive integer into parts with colors are called colored partitions." As an example, if 1, 2 and 3 are assigned with two colors, then possible partitions of 3 are $3_{i}, 3_{v}, 2_{v}+$ $1_{v}, 2_{i}+1_{i}, 2_{i}+1_{v}, 2_{v}+1_{i}, 1_{i}+1_{i}+1_{i}, 1_{v}+1_{v}+1_{v}$, $1_{i}+1_{i}+1_{v}$ and $1_{i}+1_{v}+1_{v}$, where we used the indices $i$ (indigo) and $v$ (violet) to differentiate two colors of 1,2 and 3 . Also, the generating function for the number of partitions of $n$ is defined as

$\frac{1}{\left(q^{a} ; q^{b}\right)_{\infty}^{k}}$,

with $k$ colors, and all the parts are congruent to $a(\bmod b)$.

Theorem 1 If $\alpha(n)$ represent the number of partitions of $n$ being divided into parts that are congruent to $\pm 1, \pm 3$ modulo 10 with six colors, $\pm 2, \pm 4$ modulo 10 with four colors and +5 with eight colors, respectively. If $\beta(n)$ is chosen to represent the number of partitions of $n$ into many parts that are congruent to $\pm 1, \pm 3$ modulo 10 with three colors, $\pm 2, \pm 4$ modulo 10 with two colors and +5 with four colors, respectively. If $\gamma(n)$ indicates the number of partitions of $n$ being split into parts congruent to $\pm 1, \pm 3$ modulo 10 with nine colors, $\pm 2, \pm 4$ modulo 10 with two colors and +5 with four colors, respectively. Then, the following relation holds true:

$20 \alpha(n-2)+9 \beta(n-1)-\gamma(n)=0, \quad n \geq 2$.

Proof Using (17) in (3), then dividing throughout by $f_{1}^{9} f_{2}^{7} f_{5}^{7}$ $f_{10}^{9}$ and simplifying to the common base $q^{10}$, we have

$$
\begin{aligned}
1 & +\frac{20 q^{2}}{\left(q_{6}^{1}, q_{4}^{2}, q_{6}^{3}, q_{4}^{4}, q_{8}^{5}, q_{4}^{6}, q_{6}^{7}, q_{4}^{8}, q_{6}^{9} ; q^{10}\right)_{\infty}} \\
& +\frac{9 q}{\left(q_{3}^{1}, q_{2}^{2}, q_{3}^{3}, q_{2}^{4}, q_{4}^{5}, q_{2}^{6}, q_{3}^{7}, q_{2}^{8}, q_{3}^{9} ; q^{10}\right)_{\infty}} \\
& -\frac{1}{\left(q_{9}^{1}, q_{2}^{2}, q_{9}^{3}, q_{2}^{4}, q_{4}^{5}, q_{2}^{6}, q_{9}^{7}, q_{2}^{8}, q_{9}^{9} ; q^{10}\right)_{\infty}}=0 .
\end{aligned}
$$

For simplicity, we write

$$
\left(q^{ \pm a} ; q^{b}\right)_{\infty}:=\left(q_{k}^{a}, q_{k}^{b-a} ; q^{b}\right)_{\infty} \quad a<b, \quad a, b \in \mathbb{Z}^{+} .
$$

Using the above identity in (25), we obtain

$$
\begin{aligned}
1 & +\frac{20 q^{2}}{\left(q_{6}^{ \pm 1}, q_{4}^{ \pm 2}, q_{6}^{ \pm 3}, q_{4}^{ \pm 4}, q_{8}^{+5} ; q^{10}\right)_{\infty}} \\
& +\frac{9 q}{\left(q_{3}^{ \pm 1}, q_{2}^{ \pm 2}, q_{3}^{ \pm 3}, q_{2}^{ \pm 4}, q_{4}^{+5} ; q^{10}\right)_{\infty}} \\
& -\frac{1}{\left(q_{9}^{ \pm 1}, q_{2}^{ \pm 2}, q_{9}^{ \pm 3}, q_{2}^{ \pm 4}, q_{4}^{+5} ; q^{10}\right)_{\infty}}=0 .
\end{aligned}
$$

On using (24) in (26), we obtain the three generating functions, namely $\alpha(n), \beta(n)$ and $\gamma(n)$, respectively. Now (26) reduces to

$1+20 q^{2} \sum_{n=0}^{\infty} \alpha(n) q^{n}+9 q \sum_{n=0}^{\infty} \beta(n) q^{n}-\sum_{n=0}^{\infty} \gamma(n) q^{n}=0$,

where we set $\alpha(0)=\beta(0)=\gamma(0)=1$. Extracting the coefficients of $q^{n}$, we obtain the required result.

For $n=2$, the following table verifies the above theorem.

$\begin{array}{ll}\alpha(0)=1: & \\ \beta(1)=3: & 1_{r}, 1_{g}, 1_{y} \\ \gamma(2)=47: & 1_{r}+1_{r}, 1_{g}+1_{g}, \text { and } 7 \text { others of the same type. } \\ & 1_{r}+1_{g}, 1_{g}+1_{y}, \text { and } 34 \text { others of the same type. } \\ & 2_{r}, 2_{g} .\end{array}$

Acknowledgements The authors thank anonymous referee for valuable suggestions and comments.

Funding Open access funding provided by Manipal Academy of Higher Education, Manipal.

\section{Declarations}

Conflict of interest The authors declare that they have no conflict of interest.

Ethical approval This study does not involve any human participants or animals performed by any of the authors. 
Open Access This article is licensed under a Creative Commons Attribution 4.0 International License, which permits use, sharing, adaptation, distribution and reproduction in any medium or format, as long as you give appropriate credit to the original author(s) and the source, provide a link to the Creative Commons licence, and indicate if changes were made. The images or other third party material in this article are included in the article's Creative Commons licence, unless indicated otherwise in a credit line to the material. If material is not included in the article's Creative Commons licence and your intended use is not permitted by statutory regulation or exceeds the permitted use, you will need to obtain permission directly from the copyright holder. To view a copy of this licence, visit http://creativecomm ons.org/licenses/by/4.0/.

\section{References}

Adiga C, Naika MSM, Shivashankara K (2002) On some $P-Q$ etafunction identities of Ramanujan. Indian J Math 44(3):253-267

Adiga C, Kim T, Naika MSM, Madhusudhan HS (2004) On Ramanujan's cubic continued fraction and explicit evaluations of thetafunctions. Indian J Pure Appl Math 35(9):1047-1062

Adiga C, Bulkhali NAS, Ranganatha D, Srivastava HM (2016) Some new modular relations for the Rogers-Ramanujan type functions of order eleven with applications to partitions. J Number Theory 158:281-297

Baruah ND (2002) Modular equations for Ramanujan's cubic continued fraction. J Math Anal Appl 268(1):244-255

Baruah ND (2003) On some of Ramanujan's Schlafli-type mixed modular equations. J. Number Theory 100(2):270-294

Berndt BC (1991) Ramanujan's Notebooks. Springer-Verlag, New York, Part III
Berndt BC (1996) Ramanujan's Notebooks. Springer-Verlag, New York, Part IV

Cao Z (2010) On Somos's dissection identities. J math Anal Appl 365:659-667

Naika MSM (2006) A note on cubic modular equations of degree two. Tamsui Oxf J Math Sci 22(1):1-8

Ramanujan S (1957) Notebooks (2 Volumes). Tata Institute of Fundamental Research, Bombay

Ramanujan S (1988) The Lost Notebook and other unpublished papers. Narosa, New Delhi

Saikia N (2011) Modular identities and explicit values of a continued fraction of order twelve. JP J Algebra Number Theory Appl $22: 127-154$

Somos M Personal communication

Srivatsa Kumar BR, Anu Radha D (2018) Somos's theta-function identities of level 10. Turkish J Math 42:763-773

Srivatsa kumar, B.R., Rajanna, K.R., Narendra, R., (2019) New ThetaFunction Identities of Level 6 in the Spirit of Ramanujan. Math Notes 106(6):922-929

Vasuki KR, Sreeramamurthy TG (2005) A note on $P-Q$ modular equations. Tamsui Oxf J Math Sci 21(2):109-120

Vasuki KR (2006) On some of Ramanujan's $P-Q$ modular equations. J Indian Math Soc 73(3-4):131-143

Vasuki KR, Veeresha RG (2017) On Somos's theta-function identities of level 14. Ramanujan J 42:131-144

Yi J (2004) Theta-function identities and the explicit formulas for thetafunction and their applications. J Math Anal Appl 292:381-400

Yuttanan B (2012) New modular equations in the spirit of Ramanujan. Ramanujan J 29:257-272

Publisher's Note Springer Nature remains neutral with regard to jurisdictional claims in published maps and institutional affiliations. 